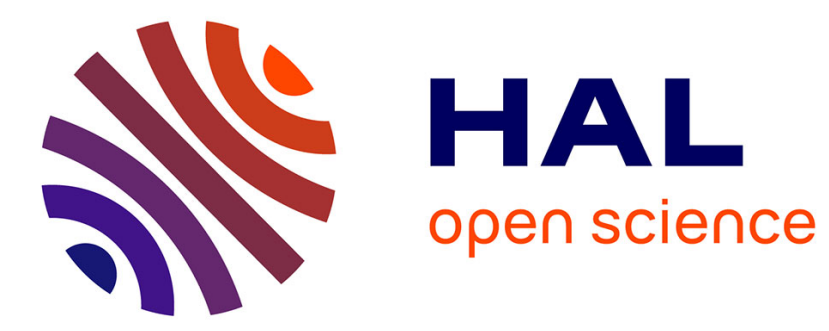

\title{
Hybrid recursive regularized thermal lattice Boltzmann model for high subsonic compressible flows
}

\author{
Yongliang Feng, Pierre Boivin, Jérome Jacob, Pierre Sagaut
}

\section{To cite this version:}

Yongliang Feng, Pierre Boivin, Jérome Jacob, Pierre Sagaut. Hybrid recursive regularized thermal lattice Boltzmann model for high subsonic compressible flows. Journal of Computational Physics, 2019, 394, pp.82-99. hal-02142837

\section{HAL Id: hal-02142837 \\ https://hal.science/hal-02142837}

Submitted on 28 May 2019

HAL is a multi-disciplinary open access archive for the deposit and dissemination of scientific research documents, whether they are published or not. The documents may come from teaching and research institutions in France or abroad, or from public or private research centers.
L'archive ouverte pluridisciplinaire HAL, est destinée au dépôt et à la diffusion de documents scientifiques de niveau recherche, publiés ou non, émanant des établissements d'enseignement et de recherche français ou étrangers, des laboratoires publics ou privés. 


\title{
Hybrid recursive regularized thermal lattice Boltzmann model for high subsonic compressible flows
}

\author{
Yongliang Feng ${ }^{\mathrm{a}}$, Pierre Boivin ${ }^{\mathrm{a}}$, Jérôme $\mathrm{Jacob}^{\mathrm{a}}$, Pierre Sagaut ${ }^{\mathrm{a}, *}$ \\ ${ }^{a}$ Aix Marseille Univ, CNRS, Centrale Marseille, M2P2, Marseille, France
}

\begin{abstract}
A thermal lattice Boltzmann model with a hybrid recursive regularization (HRR) collision operator is developed on standard lattices for simulation of subsonic and sonic compressible flows without shock. The approach is hybrid: mass and momentum conservation equations are solved using a lattice Boltzmann solver, while the energy conservation is solved under entropy form with a finite volume solver. The defect of Galilean invariance related to Mach number is corrected by the third order equilibrium distribution function, supplemented by an additional correcting term and hybrid recursive regularization. The proposed approach is assessed considering the simulation of i) an isentropic vortex convection, ii) a two dimensional acoustic pulse and iii) non-isothermal Gaussian pulse with Ma number in range of 0 to 1. Numerical simulations demonstrate that the flaw in Galilean invariance is effectively eliminated by the compressible HRR model. At last, the compressible laminar flows over flat plate at Ma number of 0.3 and 0.87 , Reynolds number of $10^{5}$ are considered to validate the capture of viscous and diffusive effects.
\end{abstract}

Keywords: compressible flows, thermal lattice Boltzmann model, equation

*Corresponding author: pierre.sagaut@univ-amu.fr 
of state, Mach number, recursive regularized

\section{Introduction}

The lattice Boltzmann method (LBM) is an alternative approach to simulate fluid flows based on the Boltzmann equation. The base model is well suited for the simulation of low-Mach, athermal flows [1-3]. Due to its advantages for massively parallel computing as well as its ability to handle very complex geometries, there are significant research efforts invested in extending LBM to thermal and subsonic to supersonic applications.

In achieving that goal, the numerical stability of the Lattice-Boltzmann collision model has long been identified along the key issues [4]. The most commonly used collision model is the single time relaxation process referred to as the Bhatnagar-Gross-Krook (BGK) model [5]. In order to overcome the insufficient stability observed in the BGK model, several improved collision models with enhanced stability have been proposed, e.g. multi-relaxationtime models $[6,7]$, entropic LB models $[8,9]$, regularized BGK models [10, 11], or cumulant/cascaded LB models [12, 13].

Numerous studies have shown the capabilities of LBM in the simulation of thermal flows including scalar transport processes. A hybrid finite difference thermal model using two dimensional multiple relaxation time collision model was presented for low Mach number compressible flows [14]. As reported in [15], the hybrid finite difference thermal model can simply cancel a spurious source term in thermal lattice Boltzmann models with forcing terms. Moreover, the robustness of hybrid approach was demonstrated in simulations of highly compressible flows [16].

For the simulation of compressible flows at higher Mach numbers, how- 
ever, no clear consensus has been reached within the LB framework. The classical method requires considering high-order moments of density distribution functions to recover the macroscopic energy conservation equation for thermal lattice Boltzmann model [17], usually relying on extended-neighbour lattice set (D1Q5, D2Q37, D3Q121 etc.) leading to LB methods referred to as multi-speed models $[18,19]$. This approach, seems rather expensive for industrial applications due to the high number of lattices (which may become prohibitively high in multi-component flows).

Several attempts have also been made keeping lower-order lattices (D1Q3, D2Q9, D2Q17, D3Q19, D3Q39 etc.). A two-dimensional compressible LB model with reduction of lattice sound speed through incorporating an athermal equation of state was proposed by $\mathrm{Yu}$ and Zhao [20], in which a forcing term was added in the form of the pressure gradient calculated by D2V17 lattice nodes. Next, the LB model using reduction of sound speed [21] and hybrid finite difference entropy equation was studied in D3Q39 lattice model for transonic flows [16]. As reported in studies of Kupershtokh et al. [22], both the method of incorporating forcing terms into the lattice Boltzmann model and calculation of pressure gradient are crucial to implement an arbitrary equation of state.

For the nearest-neighbour lattice sets (D1Q3, D2Q9, D3Q15, D3Q19, D3Q27, i.e. the so called standard lattices), Házi and Kávrán [23] suggested to use a third-order Hermite polynomial expansion the equilibrium distribution functions to address acoustic problems. Dellar [24] proposed a lattice Boltzmann algorithm with Hermite-Gram-Schmidt polynomials to reduce cubic defects in Galilean invariance on standard lattices for the simulation of low-subsonic flows with uniform density. Malaspinas [25] developed a recursive regularization procedure to increase stability and accuracy of the 
lattice Boltzmann scheme for athermal compressible flows at moderate Mach numbers. For thermal compressible flows, a coupled double distribution function (DDF) lattice Boltzmann model was developed for thermal flow with correcting terms in two-dimensional space of multiple relaxation time collision model [26]. A three-dimensional DDF thermal lattice Boltzmann model with general correcting term for thermal flows with variable density was developed by Feng et al. [27]. A DDF thermal lattice Boltzmann model was extended to high-subsonic flows using finite volume approach to improve numerical stability [28].

The collision models on standard lattice has also been investigated in compressible and weakly compressible flows. An entropic thermal D2Q9 model was introduced in [29] for subsonic flows characterized by large temperature and density variations. Recently, the central moment and cascaded thermal lattice Boltzmann models with variable density was proposed for for weakly compressible flows [30,31]. Besides, the recursive regularized BGK model was proposed for high Mach number flows basing on higher-order lattice set $[32,11]$. Jacob et al. [33] then proposed a dynamic hybrid recursive regularized BGK model with self-adaptive dissipation for Large-Eddy Simulation high Reynolds number flows and Reynolds-Averaged Numercial Simulation [34], which could be generally extended to compressible flows by considering the effects of turbulent fluctuations have a striking resemblance to those of microscale (thermal) fluctuations in laminar flows $[35,36]$.

Due to its intrinsic low dissipation, high fidelity and simplicity compared with the conventional finite volume/difference approach [37, 38], it is natural to seek a stable and efficient LB model able to simulate fully compressible flows on standard lattices. The objective of this paper is to present a new thermal lattice Boltzmann model relying on the nearest-neighbour lattice 
structures with a hybrid recursive regularized BGK collision model for subsonic to sonic smooth compressible flows. It is organized as follows: Section 2 presents the governing equations including the energy conservation in entropy form; Section 3 presents the thermal lattice Botlzmann method with a hybrid recursive regularized BGK collision model along with the numerical algorithm; In section 4, simulation of an isentropic vortex convection, a two dimensional acoustic pulse and non-isothermal Gaussian pulse with Ma number between 0 and 1 and a compressible laminar flow over flat plate at $M a=0.87$ are computed as validation and application. Finally, Section 5 draws conclusions and perspectives.

\section{Governing equations}

The mass and momentum conservation equations governing compressible flows read

$$
\begin{gathered}
\frac{\partial \rho}{\partial t}+\nabla \cdot(\rho \boldsymbol{u})=0 \\
\frac{\partial \rho \boldsymbol{u}}{\partial t}+\nabla \cdot(\rho \boldsymbol{u u})=-\nabla p+\nabla \cdot \boldsymbol{\tau}
\end{gathered}
$$

where $\rho, \boldsymbol{u}, p$ are the density, velocity, pressure, respectively. The viscous stress tensor $\boldsymbol{\tau}$ is given by

$$
\boldsymbol{\tau}=\mu\left[\nabla \boldsymbol{u}+(\nabla \boldsymbol{u})^{T}-\frac{2}{3}(\nabla \cdot \boldsymbol{u}) \boldsymbol{I}\right]
$$

where $\mu$ is the fluid dynamic viscosity and $\boldsymbol{I}$ is identity matrix.

The total energy conservation can be expressed as

$$
\frac{\partial \rho E}{\partial t}+\nabla \cdot[\boldsymbol{u}(\rho E+p)]=\nabla \cdot(\lambda \nabla T)+\nabla \cdot(\boldsymbol{u} \cdot \boldsymbol{\tau}),
$$

$\lambda$ is heat conductivity. Total energy $E=e+\|\boldsymbol{u}\|^{2} / 2$ is the sum of internal and kinetic energies. 
Alternatively, the energy conservation (3) can be expressed in terms of entropy as

$$
\frac{\partial s}{\partial t}+\boldsymbol{u} \cdot \nabla s=\frac{1}{\rho T} \nabla \cdot(\lambda \nabla T)+\frac{1}{\rho T} \boldsymbol{\tau}: \nabla \boldsymbol{u} .
$$

Finally, a thermodynamic closure is needed, relating the internal energy $e$ (or entropy $s$ ) with the pressure $p$ and volume mass $\rho$ through the temperature. Albeit not restricted to this equation of state, the perfect gas closure was adopted for this work, but the model is compatible of more complex closures. In the following,

$$
p=\rho . r . T, \quad e=C_{v} \cdot T+q, \quad s=C_{v} \ln \left(\frac{T}{\rho^{\gamma-1}}\right)+q^{\prime},
$$

where $C_{v}$ is the mass specific heat capacity at constant volume, assumed to be constant, $r=R / W$ is the ratio of the perfect gas constant to the gas molecular weight, $\gamma=C_{p} / C_{v}$ is the specific ratio, and $q$ and $q^{\prime}$ are references which can be set to zero for a mono-constituent gas.

\section{Hybrid recursive regularized thermal LB model}

This section presents the model we propose to solve the governing equations (1). A hybrid recursive regularized thermal LB model relying on the third order Hermite expansion will be developed incorporating a finite volume approach for solving entropy equation.

\subsection{Thermal LB model using the 3rd order Hermite expansion}

The lattice Boltzmann equation (LBE) can be derived from the Boltzmann equation in the velocity space. It can be written as follows

$$
\frac{\partial f}{\partial t}+\boldsymbol{\xi} \cdot \frac{\partial f}{\partial \boldsymbol{x}}=\Omega_{f}
$$


where $f$ denotes the density distribution function, $\boldsymbol{\xi}$ is the particle velocity and $\Omega_{f}$ is the collision operator. The macroscopic quantities, e.g., the density $\rho$, mean velocity $\boldsymbol{u}$ can be obtained from the distribution function as

$$
\begin{aligned}
\rho & =\int f d \boldsymbol{\xi}, \\
\rho \boldsymbol{u} & =\int \boldsymbol{\xi} f d \boldsymbol{\xi} .
\end{aligned}
$$

A single-relaxation-time collision operator, Bhatnagar-Gross-Krook (BGK) $[5]$,

$$
\Omega_{f}=-\frac{1}{\tau}\left(f-f^{e q}\right)
$$

is used as a basis to develop the proposed method, where $\tau$ is relaxation time. The equilibrium distribution function $f^{e q}$ can be derived as

$$
f^{e q}=\rho\left(\frac{1}{2 \pi r T}\right)^{\frac{D}{2}} \exp \left[\frac{(\boldsymbol{\xi}-\boldsymbol{u})^{2}}{2 r T}\right]
$$

where $r$ is the gas constant, $D$ is the number of spatial dimension and $T$ represents temperature.

In most LB models based on nearest neighbor lattices (D1Q3, D2Q9, D3Q27, etc.), the density distribution function is approximated through a second-order Hermite polynomials expansion, leading to discrete equilibrium distribution functions $f_{i}^{e q}$ as

$$
f_{i}^{\mathrm{eq}}=w_{i}\left[\rho+\frac{c_{i \alpha}}{c_{s}^{2}} \rho u_{\alpha}+\frac{\mathcal{H}_{i \alpha \beta}^{(2)}}{2 c_{s}^{4}} \rho u_{\alpha} u_{\beta}\right]
$$

where $w_{i}$ is the $i_{t h}$ weight coefficient associated to discrete velocity $c_{i \alpha}$, the second order Hermite polynomial reads

$$
\mathcal{H}_{i \alpha \beta}^{(2)}=c_{i \alpha} c_{i \beta}-c_{s}^{2} \delta_{\alpha \beta}
$$


with $\delta_{\alpha \beta}$ being the classical Kronecker delta, and $c_{s}$ is the sound speed.

Using the second-order approximation (10) for the equilibrium function, the Boltzmann equation (6) with BGK closure can be shown to be equivalent to the weakly compressible Navier-Stokes equation closed with the following athermal equation of state : $p=\rho c_{s}^{2}$. Because of the assumption that pressure only depends on $\rho$, the athermal equation of state is clearly not suited for fully compressible flows, in which a source of heat can set a fluid in motion (or, equivalently, a disequilibrium in the density function).

To handle compressible flows through a non-isothermal equation of state (e.g. $p=\rho r T$ for ideal gas), it is then natural to consider the thermal Maxwell-Boltzmann distribution instead [17], here expanded to the third order as

$$
f_{i}^{e q}=w_{i}\left[\rho+\frac{c_{i \alpha}}{c_{s}^{2}} \rho u_{\alpha}+\frac{\mathcal{H}_{i \alpha \beta}^{(2)}}{2 c_{s}^{4}} \mathcal{A}_{\alpha \beta}^{(0)}+\frac{\mathcal{H}_{i}^{(3)}}{6 c_{s}^{6}} \mathcal{A}_{\alpha \beta \gamma}^{(0)}\right] .
$$

Indeed, the Hermite polynomials remain unaltered, the second-order given by (11) and the third-order being

$$
\mathcal{H}_{i \alpha \beta \gamma}^{(3)}=c_{i \alpha} c_{i \beta} c_{i \gamma}-c_{s}^{2}\left[c_{i} \delta\right]_{\alpha \beta \gamma}
$$

where $\left[c_{i} \delta\right]_{\alpha \beta \gamma}=c_{i \alpha} \delta_{\beta \gamma}+c_{i \beta} \delta_{\alpha \gamma}+c_{i \gamma} \delta_{\alpha \beta}$. The second and third-order terms in the expansion now include full compressiblity as $\left(p-\rho c_{s}^{2}\right)$, now allowed to non-zero values

$$
\begin{aligned}
\mathcal{A}_{\alpha \beta}^{(0)} & =\rho u_{\alpha} u_{\beta}+\left(p-\rho c_{s}^{2}\right) \delta_{\alpha \beta}, \\
\mathcal{A}_{\alpha \beta \gamma}^{(0)} & =\rho u_{\alpha} u_{\beta} u_{\gamma}+\left(p-\rho c_{s}^{2}\right)[u \delta]_{\alpha \beta \gamma},
\end{aligned}
$$

where $[u \delta]_{\alpha \beta \gamma}=u_{\alpha} \delta_{\beta \gamma}+u_{\beta} \delta_{\alpha \gamma}+u_{\gamma} \delta_{\alpha \beta}$.

The moments of the equilibrium distribution function (12) on nearest 
neighbor type lattices are then

$$
\begin{gathered}
\sum_{i} f_{i}^{e q}=\rho, \\
\sum_{i} c_{i \alpha} f_{i}^{e q}=\rho u_{\alpha}, \\
\sum_{i} c_{i \alpha} c_{i \beta} f_{i}^{e q}=p \delta_{\alpha \beta}+\rho u_{\alpha} u_{\beta}, \\
\sum_{i} c_{i \alpha} c_{i \beta} c_{i \gamma} f_{i}^{e q}=p[u \delta]_{\alpha \beta \gamma}+\rho u_{\alpha} u_{\beta} u_{\gamma}-\Psi_{\alpha \beta \gamma} .
\end{gathered}
$$

where the term $\Psi_{\alpha \beta \gamma}$ is a correction due to defect of symmetry of the nearest neighbor type lattices for the third-order moment. This deviation is intended to be corrected by introducing a correcting term $\psi_{i}$, which can be applied as a second order forcing term in lattice Boltzmann BGK equation following

$$
\begin{gathered}
\frac{\partial f_{i}}{\partial t}+c_{i \alpha} \frac{\partial f_{i}}{\partial x_{\alpha}}=\Omega_{f_{i}}+\psi_{i} \\
\psi_{i}=-w_{i} \frac{\mathcal{H}_{i \alpha \beta}}{2 c_{s}^{4}} \frac{\partial}{\partial x_{\gamma}} \Psi_{\alpha \beta \gamma}, \quad \sum_{i} c_{i \alpha} c_{i \beta} \psi_{i}=-\frac{\partial}{\partial x_{\gamma}} \Psi_{\alpha \beta \gamma}
\end{gathered}
$$

Taking into account the Chapman-Enskog multiscale technique [39] with the second order correcting term $\psi_{i}$, the compressible Navier-Stokes equations can be recovered as follows (see Appendix A for the detailed ChapmanEnskog analysis)

$$
\begin{gathered}
\frac{\partial \rho}{\partial t}+\frac{\partial}{\partial x_{\alpha}}\left(\rho u_{\alpha}\right)=0 \\
\frac{\partial}{\partial t}\left(\rho u_{\alpha}\right)+\frac{\partial}{\partial x_{\beta}}\left(\rho u_{\alpha} u_{\beta}+p \delta_{\alpha \beta}\right)=\frac{\partial}{\partial x_{\beta}}\left[\mu\left(\frac{\partial u_{\beta}}{\partial x_{\alpha}}+\frac{\partial u_{\alpha}}{\partial x_{\beta}}-\tilde{k} \frac{\partial u_{\gamma}}{\partial x_{\gamma}}\right)\right]
\end{gathered}
$$

The correcting term $\psi_{i}$ is given, e.g., in two dimensional D2Q9 as

$$
\psi_{i}=\frac{w_{i}}{2 c_{s}^{4}}\left\{\mathcal{H}_{i x x} \frac{\partial}{\partial x}\left[\rho u_{x}\left(1-\frac{p}{\rho c_{s}^{2}}-u_{x}^{2}\right)\right]+\mathcal{H}_{i y y} \frac{\partial}{\partial y}\left[\rho u_{y}\left(1-\frac{p}{\rho c_{s}^{2}}-u_{y}^{2}\right)\right]\right\}
$$


and in three dimensional D3Q27 lattice model as

$$
\begin{aligned}
\psi_{i}= & \frac{w_{i}}{2 c_{s}^{4}}\left\{\mathcal{H}_{i x x} \frac{\partial}{\partial x}\left[\rho u_{x}\left(1-\frac{p}{\rho c_{s}^{2}}-u_{x}^{2}\right)\right]\right. \\
& +\mathcal{H}_{i y y} \frac{\partial}{\partial y}\left[\rho u_{y}\left(1-\frac{p}{\rho c_{s}^{2}}-u_{y}^{2}\right)\right] \\
& \left.+\mathcal{H}_{i z z} \frac{\partial}{\partial z}\left[\rho u_{z}\left(1-\frac{p}{\rho c_{s}^{2}}-u_{z}^{2}\right)\right]\right\},
\end{aligned}
$$

The correcting term $\psi_{i}$ in D3Q19 lattice is derived in Appendix B.

\subsection{Hybrid recursive regularized collision model}

Regularization procedures were proposed as to improve the numerical stability of BGK collision models for lattice Boltzmann methods [10, 25, 33]. They play the role of a filter on spurious ghost moments of the numerical scheme. Practically, a regularized distribution function is introduced through recomputing the non-equilibrium parts prior to the collision step.

By including a forcing term $\psi_{i}$ in the discrete velocity Boltzmann equation (17) and integrating it along the characteristic line with the trapezoidal rule, the LB equation can be expressed as:

$$
\begin{aligned}
f_{i}\left(x_{\alpha}+c_{i \alpha} \delta_{t}, t+\delta_{t}\right)-f_{i}\left(x_{\alpha}, t\right) & =\frac{\delta_{t}}{2}\left[\Omega_{f_{i}}\left(x_{\alpha}+c_{i \alpha} \delta_{t}, t+\delta_{t}\right)+\Omega_{f_{i}}\left(x_{\alpha}, t\right)\right] \\
& +\frac{\delta_{t}}{2}\left[\psi_{i}\left(x_{\alpha}+c_{i \alpha} \delta_{t}, t+\delta_{t}\right)+\psi_{i}\left(x_{\alpha}, t\right)(23)\right.
\end{aligned}
$$

In order to remove this implicit treatment of the collision and forcing terms, a new distribution function is introduced:

$$
\overline{f_{i}}=f_{i}-\frac{\delta_{t}}{2 \tau}\left(f_{i}^{e q}-f_{i}\right)-\frac{\delta_{t}}{2} \psi_{i}
$$

The macroscopic density and momentum can be computed as

$$
\begin{gathered}
\rho=\sum_{i} \overline{f_{i}}+\frac{\delta_{t}}{2} \psi_{i} \\
\rho u_{\alpha}=\sum_{i} c_{i \alpha} \overline{f_{i}}+\frac{\delta_{t}}{2} \sum_{i} c_{i \alpha} \psi_{i}
\end{gathered}
$$


Therefore, the LBGK equation can be expressed equivalently as

$$
\overline{f_{i}}\left(x_{\alpha}+c_{i \alpha} \delta_{t}, t+\delta_{t}\right)=f_{i}^{e q}\left(x_{\alpha}, t\right)+\left(1-\frac{1}{\bar{\tau}}\right) \overline{f_{i}^{n e q}}+\frac{\delta_{t}}{2} \psi_{i}\left(x_{\alpha}, t\right)
$$

where $\bar{\tau}=\tau / \delta_{t}+1 / 2$ and $\overline{f_{i}^{n e q}}$ represents the off-equilibrium part of the new distribution functions,

$$
\overline{f_{i}^{n e q}}=\overline{f_{i}}-f_{i}^{e q}+\frac{\delta_{t}}{2} \psi_{i}=\frac{2 \bar{\tau}}{2 \bar{\tau}-1} f_{i}^{n e q}
$$

The off-equilibrium distribution function can be also expanded using Hermite polynomials according to the Chapman-Enskog technique, leading to

$$
\overline{f_{i}^{n e q}}=\frac{2 \bar{\tau}}{2 \bar{\tau}-1} f_{i}^{n e q} \approx w_{i}\left[\frac{\mathcal{H}_{i \alpha \beta}^{(2)}}{2 c_{s}^{4}} \mathcal{A}_{\alpha \beta}^{(1)}+\frac{\mathcal{H}_{i \alpha \beta \gamma}^{(3)}}{6 c_{s}^{6}} \mathcal{A}_{\alpha \beta \gamma}^{(1)}\right]
$$

where

$$
\begin{aligned}
\mathcal{A}_{\alpha \beta}^{(1)} & =\frac{2 \bar{\tau}}{2 \bar{\tau}-1} \sum_{i} c_{i \alpha} c_{i \beta} f_{i}^{n e q}=\sum_{i} c_{i \alpha} c_{i \beta} \overline{f_{i}^{n e q}} \\
& =-\delta_{t} \bar{\tau} p\left[\frac{\partial u_{\beta}}{\partial x_{\alpha}}+\frac{\partial u_{\alpha}}{\partial x_{\beta}}-\tilde{k} \frac{\partial u_{\gamma}}{\partial x_{\gamma}} \delta_{\alpha \beta}\right] \\
\mathcal{A}_{\alpha \beta \gamma}^{(1)} & \approx u_{\alpha} \mathcal{A}_{\beta \gamma}^{(1)}+u_{\beta} \mathcal{A}_{\gamma \alpha}^{(1)}+u_{\gamma} \mathcal{A}_{\alpha \beta}^{(1)}
\end{aligned}
$$

where $\tilde{k}=\gamma-1, \gamma$ being heat specific ratio. The third order recursive regularization procedure of the thermal LB model by Eq. (30) is used to reduce the Galilean invariance and increase numerical stability, which could be derived by binomial theorem of Hermite polynomials [32, 11]. The third order off-equilibrium terms on gradient of temperature derived by Ref. [32] are not considered in this work, since they are related to contributions of higher-order heat flux terms beyond Navier-Stokes equations.

Although the higher-order recursive regularization can reduce the defect of Galilean invariance, the non-hydrodynamic modes were also introduced 
by the higher-order off-equilibrium moment. A parameterized hybrid recursive regularized procedure is proposed to suppress those non-hydrodynamic modes. According to Eq. (30), the off-equilibrium moment $\mathcal{A}_{\alpha \beta}^{(1)}$ can be alternatively reconstructed by velocity gradients which could be calculated by a finite difference operator. Thus, following [33] an explicit stabilization procedure relying on the linear combination of $\mathcal{A}_{\alpha \beta}^{(1, \mathrm{RR})}$ given by Eq. (29) and $\mathcal{A}_{\alpha \beta}^{(1, \mathrm{FD})}$ obtained by second-order finite-difference approximation of Eq. (30) is introduced, leading to the definition of the following hybrid recursive regularized collision operator

$$
\mathcal{A}_{\alpha \beta}^{(1, \mathrm{HRR})}=\sigma \mathcal{A}_{\alpha \beta}^{(1, \mathrm{RR})}+(1-\sigma) \mathcal{A}_{\alpha \beta}^{(1, \mathrm{FD})}
$$

where $\sigma \in[0,1]$ is an arbitrary weighting coefficient. $\mathcal{A}_{\alpha \beta \gamma}^{(1, \mathrm{HRR})}$ is recursively updated by re-computed off-equilibrium moment $\mathcal{A}_{\alpha \beta}^{(1, \mathrm{HRR})}$.

In order to preserve the isotropy of the scheme, a second-order isotropic central difference scheme is employed to compute the numerical gradient operator. The second-order isotropic central difference scheme is given in lattice units as

$$
\frac{\partial \phi}{\partial x_{\alpha}}=\frac{1}{c_{s}^{2}} \sum_{i} w_{i} c_{i \alpha} \phi\left(x_{\alpha}+c_{i \alpha}\right)
$$

Finally, the hybrid recursive regularization procedure for thermal compressible flow is summarized as follows:

$$
\begin{gathered}
\overline{f_{i}}\left(x_{\alpha}+c_{i \alpha} \delta_{t}, t+\delta_{t}\right)=f_{i}^{e q}\left(x_{\alpha}, t\right)+\left(1-\frac{1}{\bar{\tau}}\right) \mathcal{R}\left(\bar{f}_{i}^{n e q}\right)+\frac{\delta_{t}}{2} \psi_{i}\left(x_{\alpha}, t\right) \\
\mathcal{R}\left({\overline{f_{i}}}^{n e q}\right)=w_{i}\left[\frac{\mathcal{H}_{i \alpha \beta}^{(2)}}{2 c_{s}^{4}} \mathcal{A}_{\alpha \beta}^{(1, \mathrm{HRR})}+\frac{\mathcal{H}_{i \alpha \beta \gamma}^{(3)}}{6 c_{s}^{6}} \mathcal{A}_{\alpha \beta \gamma}^{(1, \mathrm{HRR})}\right] \\
\mathcal{A}_{\alpha \beta}^{(1, \mathrm{RR})}=\sum_{i} c_{i \alpha} c_{i \beta} \overline{f_{i}^{n e q}} \\
\overline{f_{i}^{n e q}}=\overline{f_{i}}\left(x_{\alpha}, t\right)-f_{i}^{e q}\left(x_{\alpha}, t\right)+\frac{\delta_{t}}{2} \psi_{i}\left(x_{\alpha}, t\right)
\end{gathered}
$$


In the simulations, physical and lattice units for length, time, mass and pressure are related through a reference length scale $L_{0}$, the physical reference sound speed $c_{s p 0}=\sqrt{p_{0} / \rho_{0}}$ for space and time, a physical reference pressure $p_{0}$, a physical reference density $\rho_{0}$ and a lattice reference density $\rho_{l 0}$. Accordingly, the physical variables can be converted from the quantities in lattice units ( $l$ subscript) as

$$
\begin{aligned}
\delta_{x}=L_{0} / N_{n}, & \delta_{t}=\delta_{x} \cdot \frac{c_{s}}{c_{s p 0}}, \quad t=N_{t} \cdot \delta_{t} \\
x=N_{i} \cdot \delta_{x}, & \rho=\rho_{l} \cdot \frac{\rho_{0}}{\rho_{l 0}}, \quad u=u_{l} \cdot \frac{\delta_{x}}{\delta_{t}}, \\
\mu=\mu_{l} \frac{\delta_{x}^{2}}{\delta_{t}}, & p=\frac{\rho_{0}}{\rho_{l 0}} \cdot \frac{\delta_{x}^{2}}{\delta_{t}^{2}} .
\end{aligned}
$$

The physical reference sound speed $c_{s p 0}$ could be set to the far-field or free-stream sound speed in compressible flows, it is more convenient to keep it as a free parameter. This allows to freely accelerate the convergence rate, by varying the time step in the same manner of Courant-Friedrichs-Lewy (CFL) number for conventional Navier-Stokes solvers. Taking ideal gas as an example, the physical sound speed for ideal gas can be computed in the LB model as follows

$$
c_{s p}=\sqrt{\partial p / \partial \rho_{\mid s}}=\sqrt{\gamma R_{g} T}=c_{s p 0} \sqrt{\gamma T / T_{0}}
$$

where $T_{0}$ is physical reference temperature corresponding to $p_{0}$.

\subsection{Finite volume method for entropy equation}

In the hybrid lattice Boltzmann method presented in this section, the entropy based energy conservation equation is solved separately using a finite volume technique. The entropy equation with viscous heat dissipation term is given as follow

$$
\frac{\partial s}{\partial t}+u_{\alpha} \frac{\partial s}{\partial x_{\alpha}}=\frac{1}{\rho T} \frac{\partial}{\partial x_{\alpha}}\left(\lambda \frac{\partial T}{\partial x_{\alpha}}\right)+\frac{\dot{\Phi}}{\rho T} .
$$


where $\dot{\Phi}$ represents the term of viscous dissipation. An explicit second-order Runge-Kutta scheme is adopted as temporal integration, which is given as

$$
\begin{aligned}
& s^{(n+1 / 2)}=s^{(n)}+\frac{\delta_{t}}{2} \operatorname{RHS}\left[s^{(n)}\right] \\
& s^{(n+1)}=s^{(n)}+\delta_{t} \operatorname{RHS}\left[s^{(n+1 / 2)}\right]
\end{aligned}
$$

The convective flux is constructed using MUSCL scheme, while the classical second-order central difference scheme is adopted for the diffusion term and term of viscous dissipation. The third order MUSCL scheme [40] adopted in this study is given as follows:

$$
u \frac{\partial \phi}{\partial x}=u_{i} \frac{\phi_{i+1 / 2}-\phi_{i-1 / 2}}{\delta_{x}}
$$

$\phi_{i+1 / 2}$ for instance, can be given as

$$
\phi_{i+\frac{1}{2}}=\left\{\begin{array}{l}
\phi_{i+\frac{1}{2}}^{L}, u_{i}>0 \\
\phi_{i+\frac{1}{2}}^{R}, u_{i} \leq 0
\end{array}\right.
$$

and

$$
\begin{aligned}
& \phi_{i+\frac{1}{2}}^{L}=\phi_{i}+\frac{\psi\left(r_{i}\right)}{4}\left[(1-\kappa) \delta \phi_{i-\frac{1}{2}}+(1+\kappa) \delta \phi_{i+\frac{1}{2}}\right], \\
& \phi_{i+\frac{1}{2}}^{R}=\phi_{i+1}-\frac{\psi\left(r_{i+1}\right)}{4}\left[(1-\kappa) \delta \phi_{i+\frac{3}{2}}+(1+\kappa) \delta \phi_{i+\frac{1}{2}}\right],
\end{aligned}
$$

where $\kappa=1 / 3$, and,

$$
\begin{aligned}
& \delta \phi_{i+\frac{1}{2}}=\left(\phi_{i+1}-\phi_{i}\right), \delta \phi_{i-\frac{1}{2}}=\left(\phi_{i}-\phi_{i-1}\right), \\
& \delta \phi_{i+\frac{3}{2}}=\left(\phi_{i+2}-\phi_{i+1}\right), \delta \phi_{i-\frac{3}{2}}=\left(\phi_{i-1}-\phi_{i-2}\right), \\
& r_{i}=\frac{\phi_{i}-\phi_{i-1}}{\phi_{i+1}-\phi_{i}}
\end{aligned}
$$

where $i$ represents index of grid rather than lattice discrete velocity. The van Albada limiter function $\varphi(r)=2 r /\left(1+r^{2}\right)$ is used to avoid spurious oscillations [41]. The coupling between the LB and finite volume (FV) solvers is illustrated in Fig.1, along with the relevant data exchanges. 


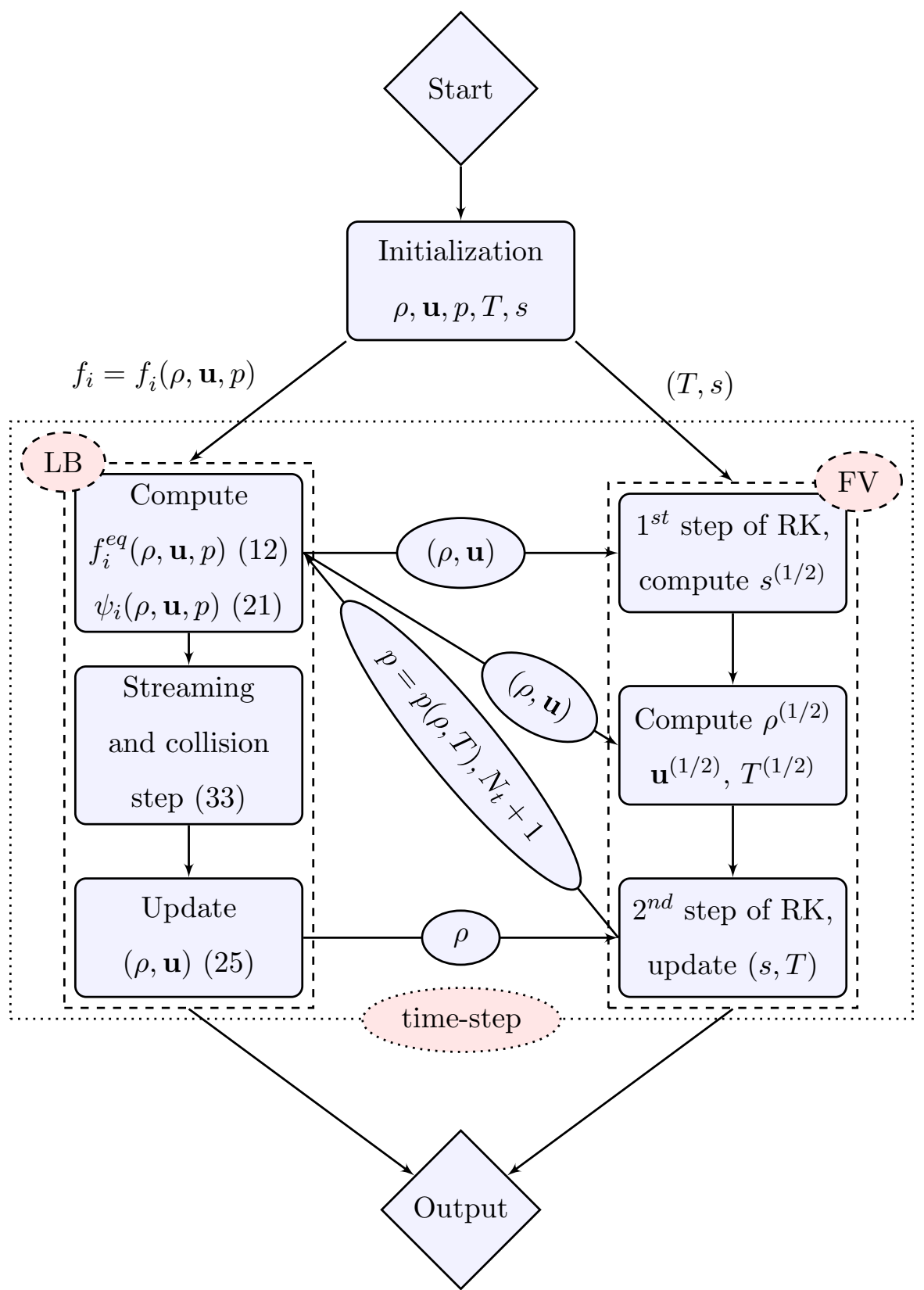

Figure 1: The algorithm proposed consists of a Lattice-Boltzmann (LB) solver coupled with a classical Finite Volume (FV) solver. Data exchanges between the two solvers are clearly identified. 


\subsection{Implementation of boundary conditions}

The boundary conditions of density distribution function are implemented by a finite difference reconstruction approach along with the hybrid regularization procedure [10].

1. First, the macroscopic velocities $\rho, \boldsymbol{u}$ and $p$ on the boundary nodes are computed, following boundary conditions commonly used in NavierStokes solvers. The entropy $s$ and temperature $T$ are then calculated by the thermodynamic closure and equation of state.

2. Next, the shear stress tensor is computed using the velocity gradients on boundary nodes, which are computed on these nodes using a first order biased finite-difference scheme.

3. Then, the density distribution function is reconstructed using $\sigma=0$ in hybrid regularization in which the off-equilibrium moment is reconstructed as follows

$$
\begin{aligned}
\mathcal{A}_{\alpha \beta}^{(1)} & \approx-\bar{\tau} \delta_{t} p\left(\frac{\partial u_{\alpha}}{\partial x_{\beta}}+\frac{\partial u_{\beta}}{\partial x_{\alpha}}+\tilde{k} \frac{\partial u_{\gamma}}{\partial x_{\gamma}} \delta_{\alpha \beta}\right), \\
\mathcal{A}_{\alpha \beta \gamma}^{(1)} & =u_{\alpha} \mathcal{A}_{\beta \gamma}^{(1)}+u_{\beta} \mathcal{A}_{\gamma \alpha}^{(1)}+u_{\gamma} \mathcal{A}_{\alpha \beta}^{(1)},
\end{aligned}
$$

In the following, prescription of macroscopic variables (step 1 above), is done according to the boundary condition.

- For a velocity inlet, $\rho, \boldsymbol{u}, p$ are set as Dirichlet boundary conditions.

- For a pressure outlet, $p$ is set as Dirichlet condition; $\rho$ and $\boldsymbol{u}$ are extrapolated enforcing a zero-gradient condition.

- For a no-slip wall, $\boldsymbol{u}=0$ is set as Dirichlet condition, and $\rho$ and $p$ are extrapolated enforcing a zero-gradient condition. 


\section{Results and discussion}

Since the base HRR LB model has already been assessed for simulation of thermal flows in low Mach number limit $[27,42,36]$, the present compressible HRR LB model is assessed considering three cases dealing with compressible subsonic to sonic shock-free flows:

1. isentropic vortex convection by a uniform flow,

2. acoustic pulse,

3. flow over a flat plate.

The first two configurations are treated as quasi-inviscid, with a non-dimensional viscosity $\mu=10^{-15}$, as to test the model in extreme conditions. For the third configuration, variable viscosity is assumed.

For the D2Q9 lattice, the microscopic discrete velocity $c_{i \alpha}$ is given by

$$
c_{i \alpha}= \begin{cases}(0,0) & i=0 \\ ( \pm 1,0),(0, \pm 1) & i=1-4 \\ ( \pm 1, \pm 1) & i=5-8\end{cases}
$$

the weights $w_{i}=4 / 9, i=0 ; w_{i}=1 / 9, i=1,2,3,4 ; w_{i}=1 / 36, i=5,6,7,8$ and the lattice reference sound speed $c_{s}$ being $\sqrt{1 / 3}$.

\subsection{Isentropic vortex convection by a uniform flow}

This is one of the few exact analytical solutions of the compressible Euler equations. This test case is related to the convection of an isentropic vortex by an inviscid uniform flow at free-stream Mach number $M a \approx 0.42$ $\left(u_{\infty}=0.5, \gamma=1.4\right)$. It is used to show the ability of numerical schemes to accurately capture vortical flows. The free-stream conditions and perturbations added to the free-stream is initialized in a computational domain 
of size $[0,10] \times[0,10]$. There is no entropy gradient in the flow-field. The free-stream flow is given by

$$
\rho_{\infty}=1, u_{\infty}=0.5, v_{\infty}=0, p_{\infty}=1
$$

and the density and velocity perturbations are initially given as a vortex, defined as

$$
\begin{aligned}
\rho & =\left[1-\frac{(\gamma-1) b^{2}}{8 \gamma \pi^{2}} e^{1-r^{2}}\right]^{\frac{1}{\gamma-1}}, p=\rho^{\gamma}, \\
u & =u_{\infty}-\frac{b}{2 \pi} e^{\frac{1}{2}\left(1-r^{2}\right)}\left(y-y_{c}\right) \\
v & =v_{\infty}+\frac{b}{2 \pi} e^{\frac{1}{2}\left(1-r^{2}\right)}\left(x-x_{c}\right) .
\end{aligned}
$$

where $b=0.5$ is the vortex strength and $r=\left[\left(x-x_{c}\right)^{2}+\left(y-y_{c}\right)^{2}\right]^{1 / 2}$ is the distance from the vortex center $\left(x_{c}, y_{c}\right)=(5,5)$.

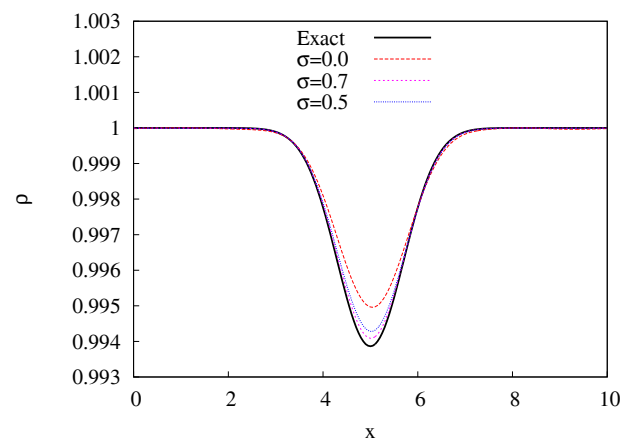

(a) Density

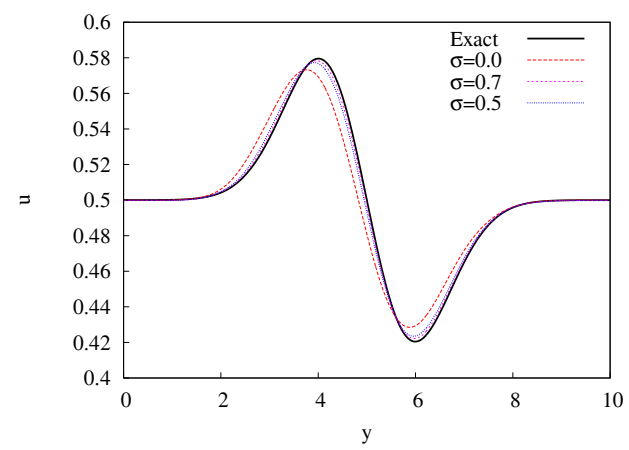

(b) Velocity

Figure 2: Profiles obtained by the LB method on isentropy vortex convection at $t=20 T$.

In our simulation, $200 \times 200$ node grids are employed with $\delta_{x}=0.05$. The time step is $\delta_{t}=\delta_{x} / \sqrt{T_{0}}$ with reference temperature $T_{0}=36.0$. An approximate inviscid condition is implemented setting the dynamic viscosity to $\mu=10^{-15}$ in the simulation, which results a relaxation time $\bar{\tau} \approx 0.5$. The 
considered values of the HRR weighting parameter are $\sigma=0.7,0.5$ and 0.0 . Periodic boundary conditions are implemented in all directions.

Figure 2 displays the distributions of density and velocities along the domain symmetry lines after 20 flow-through-time (FTT), i.e. $t=20 T$. The density along the horizontal line of symmetry is plotted in Fig. 2(a) while $\mathrm{x}$-velocity along vertical middle line is shown in Fig. 2(b). It can be observed that all results are in good agreement with the exact solution after 20 FTT. The numerical dissipation increases with decreasing HRR parameter $\sigma$ where $\sigma=0.0$ means that the off-equilibrium distribution function is completely reconstructed by the finite difference scheme.

The typical density fields obtained using different HRR parameter $\sigma$ values after $20 \mathrm{FTT}$ are presented in Fig. 3. It is found that the isotropic evolution is well preserved in the simulations except when $\sigma=0.0$. The history of the maximum of the amplitude $A$ of the density disturbance normalized by its initial value

$$
A=\frac{1-\min (\rho)}{1-\rho_{0}}
$$

is reported in Fig. 3(d). The damping rate induced by numerical dissipation is quite small. Considering both the robustness and the accuracy, the HRR parameter $\sigma=0.5$ appears to be a good trade-off for the simulation of inviscid compressible flows.

Simulations displayed in Fig. 2 were repeated varying the grid resolution $N_{x} \times N_{x}$, with $N_{x}=(25,50,100,200,400)$. The resulting profiles, reported in Figs. 4(a) and 4(b), illustrate well the grid convergence, as well as the method robustness. Further investigation of the $\mathcal{L}_{2}$ norm of these profiles, illustrated in Fig. 4(c), shows that the convergence is second order in space, a classical characteristic of LB models [3]. 


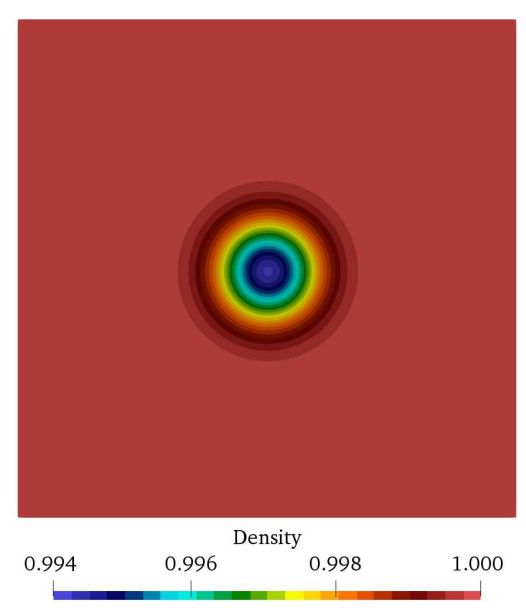

(a) $\sigma=0.5, t=20 T$



(c) $\sigma=1.0, t=20 T$

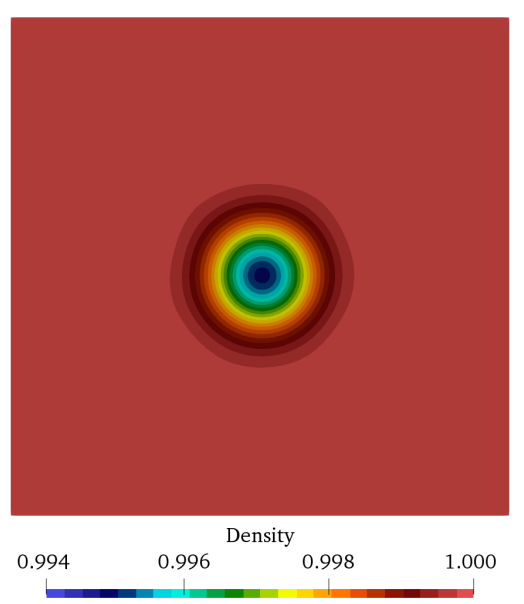

(b) $\sigma=0.0, t=20 T$

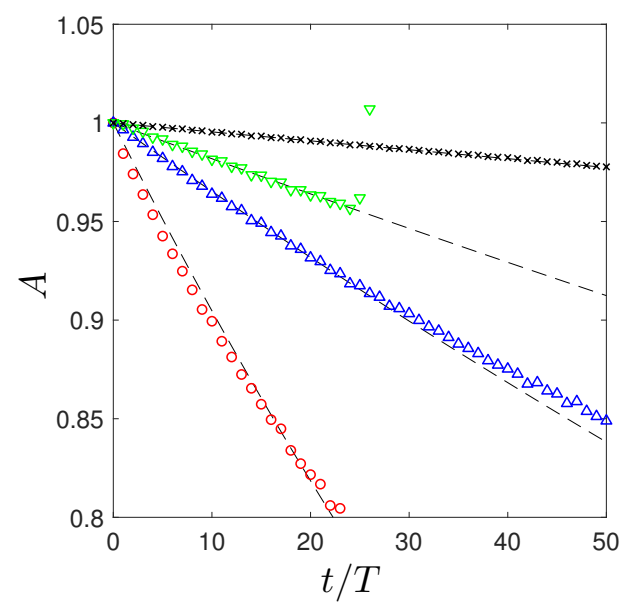

(d) Damping of the density spot with time

Figure 3: (a,b,c) Density contours after 20 periods, as obtained using different values for HRR parameter $\sigma$, on a $200 \times 200$ grid.

(d) Evolution of $A$ (46) at the center of the vortex, as obtained on a $200 \times 200$ grid: $\sigma=0$ (॰) $\sigma=0.5(\triangle), \sigma=0.7(\nabla)$, and on a $400 \times 400$ grid with $\sigma=0.5(\times$. Dashed lines are the exponential fits of the form $A(t)=e^{-t / R e^{*}}(48)$, as reported in Tab. 1. 


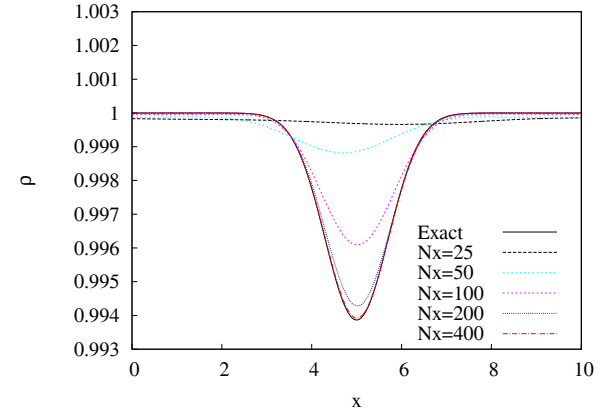

(a) Density

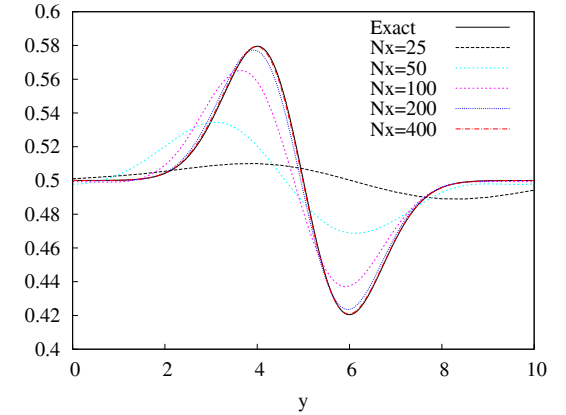

(b) Velocity

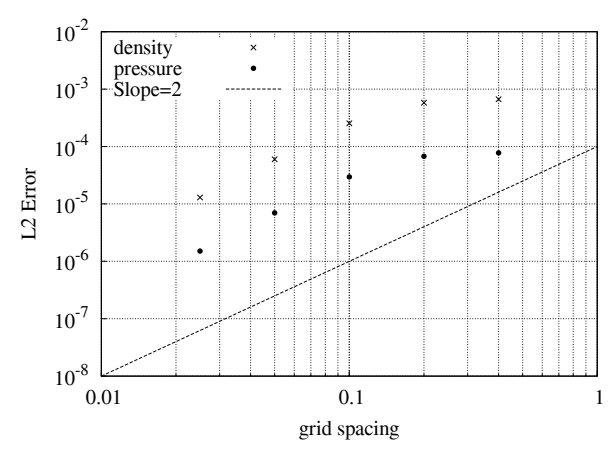

(c) $\mathcal{L}_{2}$ convergence study

Figure 4: Convergence study on the isentropic vortex convection, as in Fig. 2, repeated on $N_{x} \times N_{x}$ grids with varying $N_{x}$. 
Stability at low grid resolutions is ensured by numerical viscosity, which we shall now assess. As an approximated method, we propose to compare the classical vorticity $\omega$ decay in incompressible fluid at rest [43-45]

$$
\frac{\partial \omega}{\partial t}=\frac{\nabla \omega}{R e},
$$

where $R e$ is the Reynolds number, leading to an exponential decay of the maximum vorticity in the vortex as $e^{-t / R e}$.

Continuity in stationary compressible flows being translated in a divergence free momentum $\rho u$ field rather than divergence free velocity field $u$, it is reasonable to approximate $A(46)$ as

$$
A(t) \approx e^{-t / R e^{*}}
$$

where $R e^{*}$ corresponds to a Reynolds number associated to a numerical viscosity $\nu^{*}$. The corresponding non-dimensional values, corresponding to a reference non-dimensional length of 3 (the approximate vortex diameter) and the reference non-dimensional velocity of $u_{\infty}=0.5$ are reported in Table 1. As expected, $\nu^{*}$, given here in non-dimensional unit, decreases

\begin{tabular}{cccc} 
Resolution & $\sigma$ & $R e^{*}$ & $\nu^{*} \times 10^{5}$ \\
\hline $200 \times 200$ & 0 & 1998 & 75 \\
$200 \times 200$ & 0.5 & 5665 & 26 \\
$200 \times 200$ & 0.7 & 10910 & 14 \\
$400 \times 400$ & 0.5 & 43726 & 3.4
\end{tabular}

Table 1: Approximated numerical viscosity for the isentropic vortex convection.

both with increasing resolution and increasing $\sigma$. For further illustration, the fits obtained by using the parameters of Tab. 1 are reported in dashed lines in Fig. 3(d). 


\subsection{Two-dimensional acoustic pulse}

\subsubsection{Isothermal acoustic pulse}

Next, the compressible LB model is assessed considering benchmark problems dealing with propagation of acoustic waves, more precisely the advection of an acoustic pulse by a uniform flow. A two dimensional Gaussian acoustic pulse with free-stream velocity $U_{0}=0.0$ and $U_{0}=1.0$, i.e. at free-stream Mach number $M a=0$ and $M a=1$, is considered in this section. The initial acoustic Gaussian pulse, is given by [46]

$$
\rho=1+0.01 \exp \left(-k r^{2}\right), p=1+0.01 \exp \left(-k r^{2}\right), u=U_{0}, v=0
$$

where $r=\left[\left(x-x_{c}\right)^{2}+\left(y-y_{c}\right)^{2}\right]^{1 / 2}$ is the distance from the pulse center $\left(x_{c}, y_{c}\right)=(0,0)$. The perturbation parameter is given by $k=\ln 2 / b^{2}$, and $b=2$ is the half-width of Gaussian function. A square computational domain with size $[-20,20] \times[-20,20]$ is used, along with a $400 \times 400$ grid. Periodic boundary conditions are implemented in all directions. In order to implement the simulation of isothermal acoustics to match reference analytical solutions, the entropy equation is not solved in this case and temperature is prescribed as a constant value.

The time step is $\delta_{t}=\delta_{x} / \sqrt{T_{0}}$ with reference temperature $T_{0}=25.0$. An approximate inviscid condition is implemented setting the dynamic viscosity to $\mu=10^{-15}$, which results in a relaxation time $\bar{\tau} \approx 0.5$. Following the results of the previous test case, the HRR weighting parameter is set equal to $\sigma=0.5$

The pressure fields obtained by the LB method for the two Mach numbers at time $t=10$ are shown in Fig. 5 and 6 . Furthermore, the fluctuating velocity and pressure profiles along the horizontal centerline at time $t=$ 10 are compared to analytical solutions given in [46] in Fig. 7. A very 


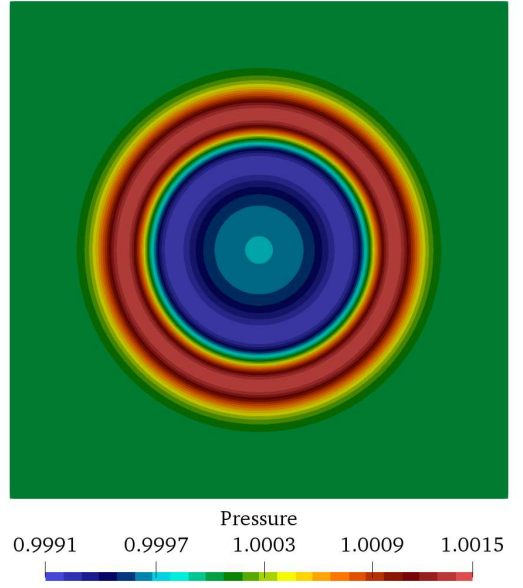

(a) $U_{0}=0$

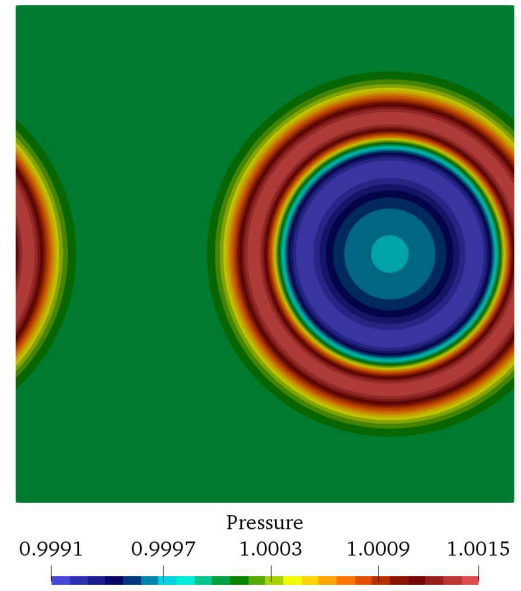

(b) $U_{0}=1$

Figure 5: Contours of pressure obtained by the LB method on acoustic pulse advection at time $t=10$.

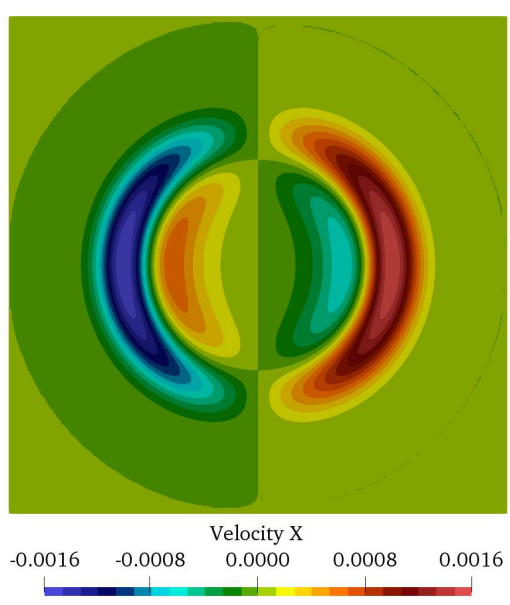

(a) $U_{0}=0$

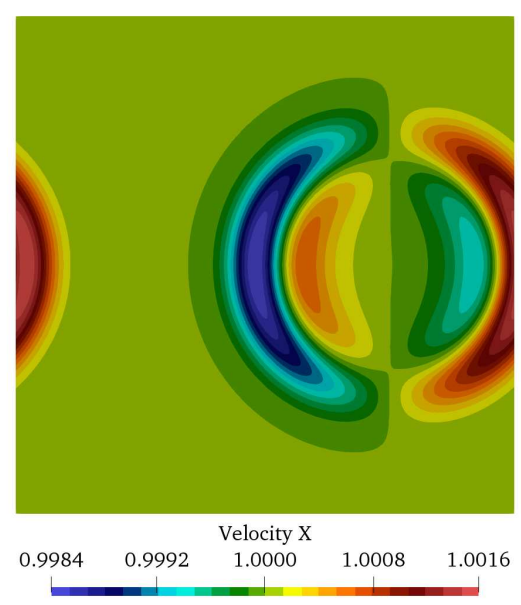

(b) $U_{0}=1$

Figure 6: Contours of velocities obtained by the LB method on acoustic pulse advection at time $t=10$. 


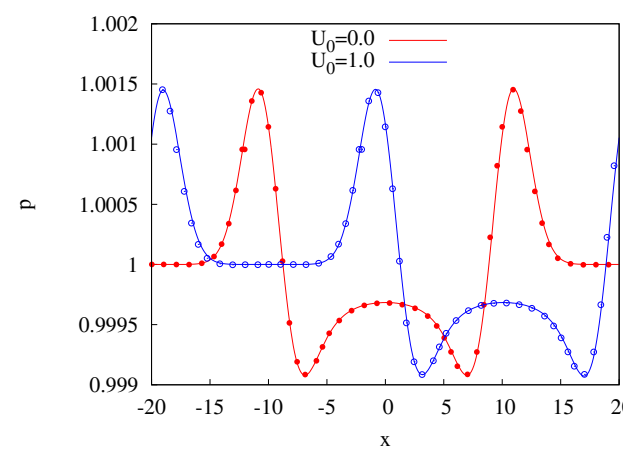

(a) pressure

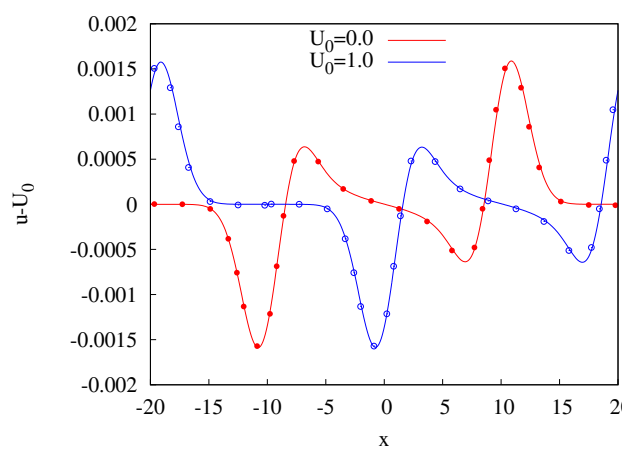

(b) velocity

Figure 7: Comparison of profiles along horizontal middle line.(line: LBM results, symbols: analytical solution)

satisfactory agreement is observed in all cases, demonstrating the capability of the present method to deal with such flows.

Comparison of the computational cost of the athermal model used in this section (e.g. without FV solver) and the proposed model shows a 75-25 cpu partition between the LB and FV solver, indicating a minimal cost of the hybrid formulation.

\subsubsection{Non-isothermal Gaussian pulse}

Next, we consider a thermal acoustic wave traveling in radial direction including temperature evolution. The aim of this test case is to verify the correct propagation speed of sound waves and coupling between velocity, pressure and temperature. The initial condition is given by:

$$
\rho=1.0, p=1+\exp \left(-k r^{2}\right), u=0, v=0
$$

where $r=\left[\left(x-x_{c}\right)^{2}+\left(y-y_{c}\right)^{2}\right]^{1 / 2}$ is the distance from the pulse center $\left(x_{c}, y_{c}\right)=(0,0)$. The perturbation parameter is taken equal to $k=40$. The computational domain size is $[-2,2] \times[-2,2]$, with periodic boundary 
conditions. The computation of the reference solution was performed using a second order TVD finite volume scheme with the Osher type flux on a radial grid consisting of $10^{4}$ cells $[47,48]$.

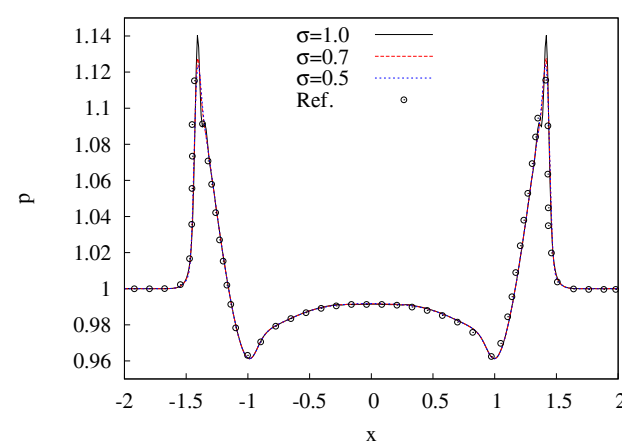

(a) pressure

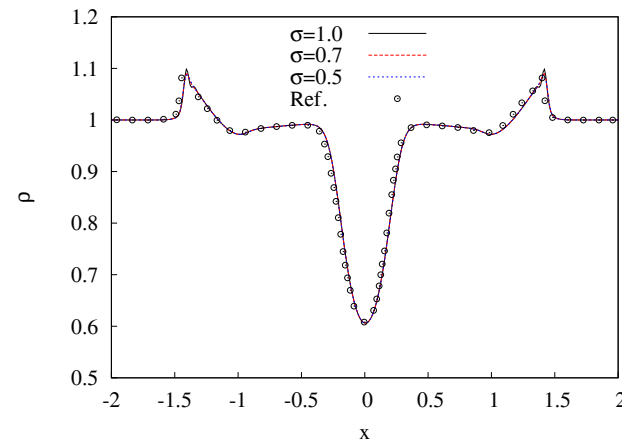

(c) density

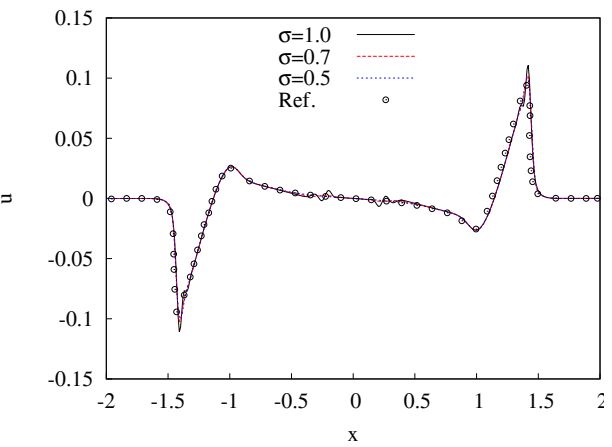

(b) velocity

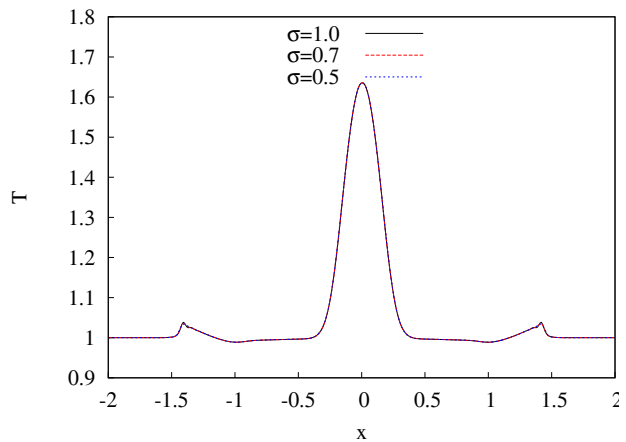

(d) temperature

Figure 8: Comparison of profiles along horizontal middle line.(line: present LBM, point: reference solution)

Figure 8 displays the profiles of pressure, horizontal velocity, density and temperature obtained by the proposed LB model using HRR parameter $\sigma=1.0,0.7$ and 0.5 at time $t_{\text {end }}=1.0$. A very good agreement with the reference solution is recovered, showing the capability of the present method to capture thermodynamic couplings and nonlinear wave propagation. 


\subsection{Compressible laminar flow over flat plate}

At last, we investigate the capability of the method to handle viscous effects addressing another typical benchmark, namely the laminar flow over a flat plate at free-stream Mach number $M a=0.3$ and $M a=0.87$. The compressible laminar flow over flat plate has been studied extensively, both analytically and numerically $[49,50]$. Here, a computational domain with size $-0.25 \leq x \leq 1,0 \leq y \leq 0.25$ is set up considering a flow at high speed subsonic $u_{\infty}$ past a thin plate of length $L($ as illustrated in Fig. 9). The boundary condition(BC) is set as the follows:

- Symmetry BC on $y=0,0.25 \leq x<0$

- No-slip wall BC on $y=0,0 \leq x \leq 1$, adiabatic BC for $M a=0.3$ while isothermal $\mathrm{BC}$ for $M a=0.87$.

- Subsonic outflow on $y=0.25$ with pressure $p=1 / \gamma$.

- Subsonic inflow on $x=0$ with density $\rho=1$, and velocity $(u, v)=$ $\left(u_{\infty}, 0\right)$.

- Subsonic outflow on $x=1$ with pressure $p=1 / \gamma$.

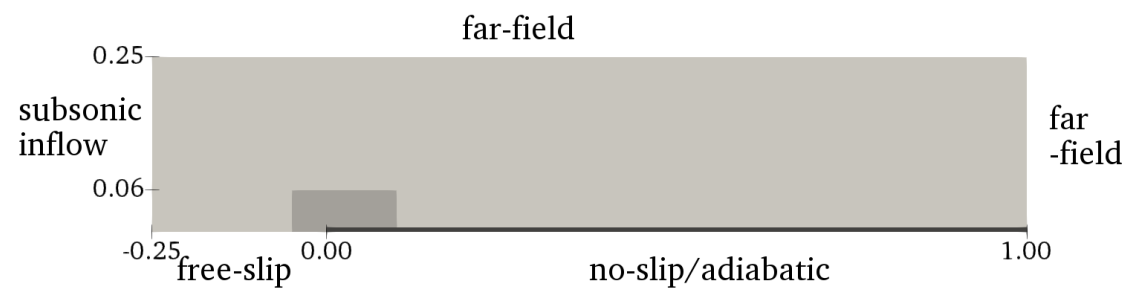

Figure 9: Sketch of the computational configuration. The darker sub-domain is used below for plotting results near the leading edge. 

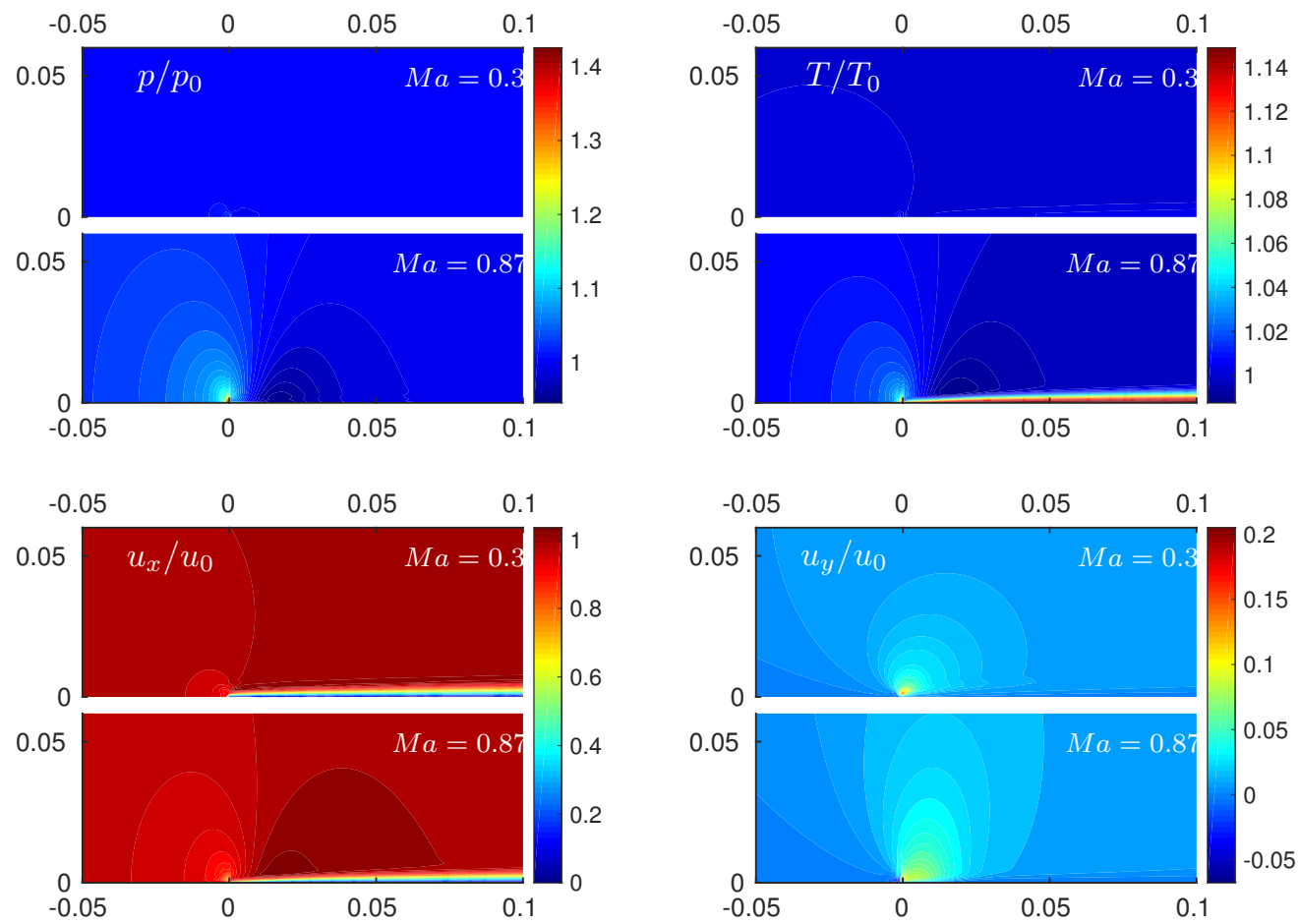

Figure 10: Contours of pressure, temperature, horizontal and vertical velocity obtained by the LB method on laminar flow over flat plate at $M a=0.3$ and $M a=0.87$ 
The Reynolds number is taken equal to $10^{5}$, leading to a steady laminar solution. A uniform dynamic viscosity is adopted at $M a=0.3$ while a variable viscosity following $\rho \mu=\rho_{\infty} \mu_{\infty}$ is used at $M a=0.87$. The entropy based energy equation is solved with Prandtl number equal to 0.72 for $M a=$ 0.3 and equal to 1.0 for $M a=0.87$. The values of $P r$ and viscosity are chosen to match those of reference similarity solutions of compressible boundary layer [51].

In simulation at $M a=0.3$, the computational grid is made of $1000 \times 200$ grid points. The spacing size is $\delta_{x}=6.25 \times 10^{-4}$ and the time step $\delta_{t}$ is set to $\delta_{x} / 6$ corresponding to a CFL number close to 0.13 . At $M a=0.87$, the computational grid has $2000 \times 400$ grid points. The spacing size is $\delta_{x}=3.125 \times 10^{-4}$ and the time step $\delta_{t}$ is set to $\delta_{x} / 8$ with a reference temperature $T_{0}$ equals 64 . The HRR parameter is set equal to $\sigma=0.9$ in all simulations.

Figure 10 displays the contours of density and horizontal velocity obtained by the present LB model at $M a=0.3$ and $M a=0.87$ in the vicinity of the plate trailing edge. It is observed that the flow is well captured by the present LB method.

Figure 11 shows the profiles of density, temperature and horizontal velocity obtained by the present LB model. In the figure, $\eta$ is the dimensionless coordinate with Illingworth transform which is defined as [51]

$$
\eta=\frac{U_{e}}{\sqrt{2 x}} \int_{0}^{y} \rho \mathrm{d} y
$$

where $U_{e}$ is velocity at outer edge and $C_{w}=1$ under condition of $\rho \mu=$ $\rho_{\infty} \mu_{\infty}$. An excellent agreement between the computed velocity fields and reference solution is observed both at $M a=0.3$ and $M a=0.87$. The skin friction predicted by the LB method on laminar flow over flat plate is also 

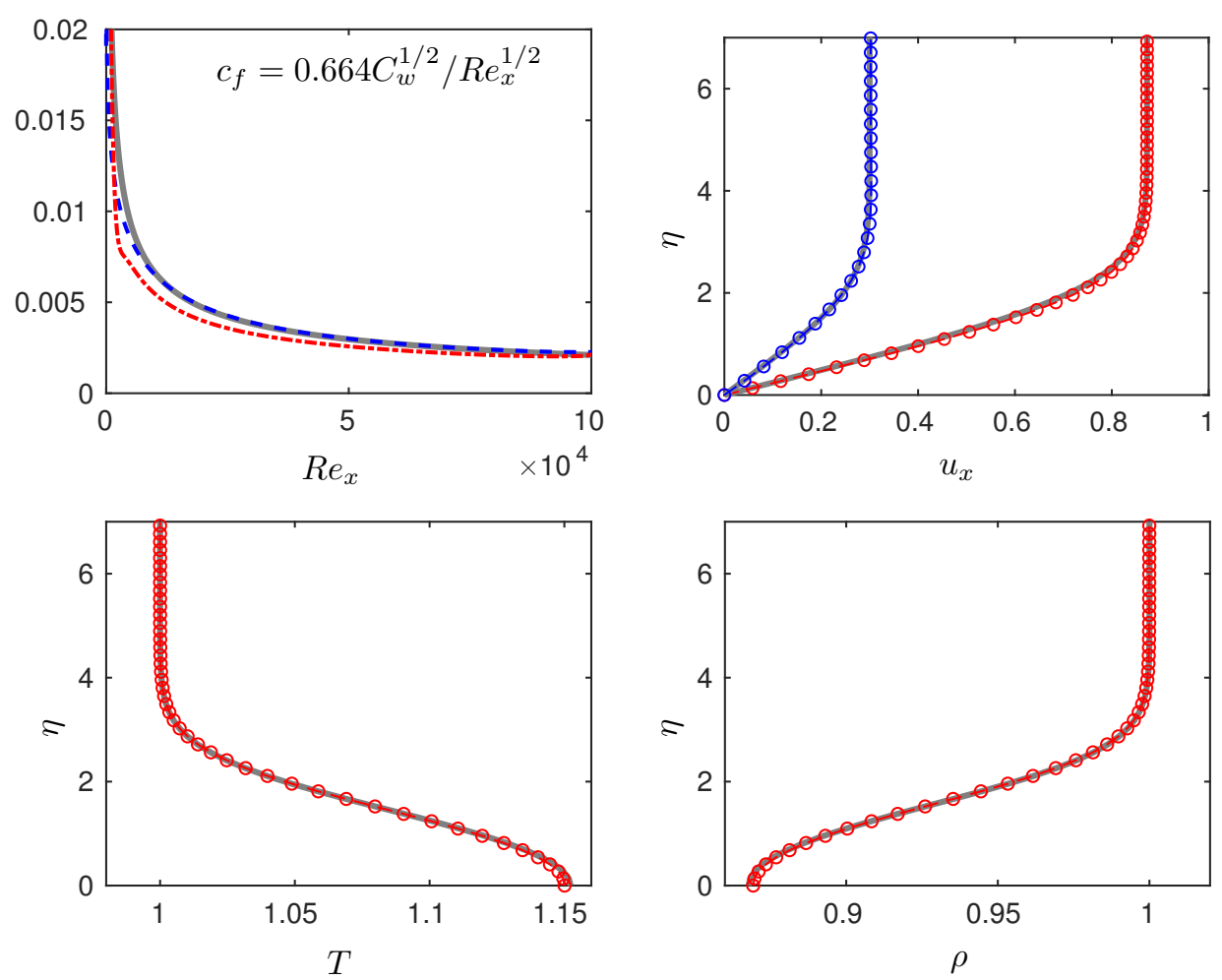

Figure 11: Profiles of the solution obtained by the LB method on laminar flow over flat plate at $M a=0.3$ (blue), and 0.87 (red). The similarity solution is plotted in black line. Top left: skin friction along the plate; top right: vertical streamwise velocity distribution in similarity coordinates; bottom left: vertical temperature distribution in similarity coordinates; bottom right: vertical temperature distribution in similarity coordinates 
well matched with Blasius solution. The maximum deviation of predicted skin friction at Mach number of 0.87 is $1.6 \%$.

In Fig. 11, the analytical solution of temperature profile for compressible flow over flat plate is given by [51]

$$
\frac{T}{T_{e}}=1+\frac{\gamma-1}{2} M a_{e}^{2}\left(1-\frac{u^{2}}{U_{e}^{2}}\right)
$$

where $T_{e}$ is temperature at outer edge. The reference solution of density is calculated by equation of state under free stream pressure. In can be observed that the excellent agreement on both temperature and density between the results obtained by the present LB method and the reference solution is observed $M a=0.87$. The coupling between fluid flow and heat transfer including viscous heat dissipation are well captured by the present LB model.

\section{Conclusions}

A thermal lattice Boltzmann model has been developed on standard lattices for the simulation of smooth subsonic to sonic compressible flows. It relies on an extension of the Hybrid Recursive Regularized LB model already proposed for low-Mach number flows.

The model is easy to implement and parallelize: both the LB and FV stencils are limited to nearest neighbors. This also leads to a cpu cost partition close to $75-25$ between the LB-FV solvers, indicating high potential regarding the cpu savings associated to the use of such model compared to classical methods.

In simulation of an isentropic vortex convection, a two dimensional acoustic pulse and non-isothermal Gaussian pulse, the influence of the HRR 
weighting parameter $\sigma$ has been intensively investigated. Numerical simulations demonstrate that the defect of Galilean invariance is effectively eliminated by $\sigma>0.5$ in the inviscid regime. Especially, the non-hydrodynamic oscillation is suppressed by hybrid regularization using $\sigma=0.5$ in simulation of non-isothermal Gaussian pulse. Excellent agreement has also been obtained for the simulation of a compressible laminar flow over flat plate at Mach number of 0.3 and 0.87 , with results matching the Blasius solution.

The low dissipation obtained with the presented model allows to envision acoustic applications in transonic flows, e.g. by accounting for the bulk viscosity.

\section{Acknowledgements}

This work was supported by the French project CLIMB, with the financial support of BPIFrance (Grant P3543-24000), in the framework of the program "Investissement d'Avenir: Calcul Intensif et Simulation Numérique". This work was granted access to the HPC resources of Aix-Marseille Université financed by the project Equip@Meso (ANR-10-EQPX-29-01) of the program Investissements d'Avenir supervised by the Agence Nationale de la Recherche. This work was performed using HPC resources from GENCITGCC/CINES (Grant 2018-A0052A07679). This work was also supported by project of ProLB software (http://www.prolb-cfd.com).

\section{Appendix A. Chapman-Enskog Analysis}

To derive macroscopic equations, the density distribution function $f_{i}$ is expanded around the $f_{i}^{e q}$ distributions as follows:

$$
f_{i}=f_{i}^{(0)}+\epsilon f_{i}^{(1)}+\epsilon^{2} f_{i}^{(2)}+\cdots
$$


with

$$
\sum_{i} f_{i}^{(n)}=0, \quad \sum_{i} c_{i \alpha} f_{i}^{(n)}=0, \quad n>0
$$

By matching the scales of $\epsilon^{1}, \epsilon^{2}$ we have

$$
\begin{gathered}
\epsilon^{1}:\left(\frac{\partial}{\partial t_{1}}+c_{i \alpha} \frac{\partial}{\partial x_{1 \alpha}}\right) f_{i}^{e q}+\frac{f_{i}^{(1)}}{\tau}=\psi_{i}^{(0)} \\
\epsilon^{2}: \frac{\partial f_{i}^{e q}}{\partial t_{2}}+\left(\frac{\partial}{\partial t_{1}}+c_{i \alpha} \frac{\partial}{\partial x_{1 \alpha}}\right) f_{i}^{(1)}+\frac{f_{i}^{(2)}}{\tau}=0
\end{gathered}
$$

Considering that the equilibrium density distribution function satisfies the velocity moment condition, one can sum Eq.(A.3)and Eq.(A.4) in the velocity phase space. The $t_{1}$ and $t_{2}$ order of the continuity equation and momentum equation can be derived as

$$
\begin{gathered}
\frac{\partial \rho}{\partial t_{1}}+\frac{\partial}{\partial x_{1 \alpha}}\left(\rho u_{\alpha}\right)=0 \\
\frac{\partial}{\partial t_{1}}\left(\rho u_{\alpha}\right)+\frac{\partial}{\partial x_{1 \beta}}\left(\rho u_{\alpha} u_{\beta}+p \delta_{\alpha \beta}\right)=0 \\
\frac{\partial \rho}{\partial t_{2}}=0 \\
\frac{\partial}{\partial t_{2}}\left(\rho u_{\alpha}\right)+\frac{\partial}{\partial x_{1 \beta}}\left(\sum_{i} c_{i \alpha} c_{i \beta} f_{i}^{(1)}\right)=0
\end{gathered}
$$

Incorporating pressure equation in convective scale as follow

$$
\frac{\partial p}{\partial t_{1}}+\frac{\partial}{\partial x_{1 \gamma}}\left(p u_{\gamma}\right)+\tilde{k} p \frac{\partial u_{\gamma}}{\partial x_{1 \gamma}}=0
$$

where $\tilde{k}=\gamma-1, \gamma$ is heat specific ratio but subscript of tensors. Combining Eq. (A.5) with Eq. (A.6), one can obtain

$$
\begin{aligned}
\frac{\partial}{\partial t_{1}}\left(\rho u_{\alpha} u_{\beta}+p \delta_{\alpha \beta}\right)= & \frac{\partial}{\partial x_{1 \gamma}} \rho u_{\alpha} u_{\beta} u_{\gamma}-u_{\alpha} \frac{\partial p}{\partial x_{1 \beta}}-u_{\beta} \frac{\partial p}{\partial x_{1 \alpha}} \\
& -\frac{\partial}{\partial x_{1 \gamma}}\left(p u_{\gamma} \delta_{\alpha \beta}\right)-\tilde{k} p \frac{\partial u_{\gamma}}{\partial x_{1 \gamma}} \delta_{\alpha \beta}
\end{aligned}
$$


Considering the correcting term $\psi_{i}$ given as

$$
\psi_{i}=-\frac{w_{i}}{2 c_{s}^{4}} \mathcal{H}_{i \alpha \beta} \frac{\partial}{\partial x_{\gamma}} \Psi_{\alpha \beta \gamma}, \quad \sum_{i} c_{i \alpha} c_{i \beta} \psi_{i}=-\frac{\partial}{\partial x_{\gamma}} \Psi_{\alpha \beta \gamma}
$$

and rewriting $f_{i}^{(1)}$ in the Eq.(A.8) with Eq.(A.3), one obtains

$$
\begin{aligned}
\sum_{i} c_{i \alpha} c_{i \beta} f_{i}^{(1)}= & -\tau \sum_{i} c_{i \alpha} c_{i \beta}\left[\left(\frac{\partial}{\partial t_{1}}+c_{i \gamma} \frac{\partial}{\partial x_{1 \gamma}}\right) f_{i}^{e q}-\psi_{i}^{(0)}\right] \\
= & -\tau\left[\frac{\partial}{\partial t_{1}}\left(\rho u_{\alpha} u_{\beta}+p \delta_{\alpha \beta}\right)+\frac{\partial}{\partial x_{\gamma}} \Psi_{\alpha \beta \gamma}\right. \\
& \left.+\frac{\partial}{\partial x_{1 \gamma}}\left(p[u \delta]_{\alpha \beta \gamma}+\rho u_{\alpha} u_{\beta} u_{\gamma}-\Psi_{\alpha \beta \gamma}\right)\right] \\
= & -\tau p\left[\frac{\partial u_{\beta}}{\partial x_{1 \alpha}}+\frac{\partial u_{\alpha}}{\partial x_{1 \beta}}-\tilde{k} \frac{\partial u_{\gamma}}{\partial x_{1 \gamma}} \delta_{\alpha \beta}\right]
\end{aligned}
$$

The following equations can be finally obtained:

$$
\begin{gathered}
\frac{\partial \rho}{\partial t}+\frac{\partial}{\partial x_{\alpha}}\left(\rho u_{\alpha}\right)=0 \\
\frac{\partial}{\partial t}\left(\rho u_{\alpha}\right)+\frac{\partial}{\partial x_{\beta}}\left(\rho u_{\alpha} u_{\beta}+p \delta_{\alpha \beta}\right)=\frac{\partial}{\partial x_{\beta}}\left[\mu\left(\frac{\partial u_{\beta}}{\partial x_{\alpha}}+\frac{\partial u_{\alpha}}{\partial x_{\beta}}-\tilde{k} \frac{\partial u_{\gamma}}{\partial x_{\gamma}} \delta_{\alpha \beta}\right)\right]
\end{gathered}
$$

\section{Appendix B. The correction term for $D 3 Q 19$ lattice set}

The D3Q19 lattice set, 3 dimensions with 19 velocities, is given by,

$$
c_{i \alpha}= \begin{cases}(0,0,0) & i=0 \\ ( \pm 1,0,0),(0, \pm 1,0),(0,0, \pm 1) & i=1-6 \\ ( \pm 1, \pm 1,0),( \pm 1,0, \pm 1),(0, \pm 1, \pm 1) & i=7-18\end{cases}
$$

and the corresponding weights are

$$
w_{i}= \begin{cases}\frac{1}{3} & i=0 \\ \frac{1}{18} & i=1-6 \\ \frac{1}{36} & i=7-18\end{cases}
$$


Considering equilibrium distribution function Eq. 12, the deviation terms can be calculated as follow

$$
\Psi_{\alpha \beta \gamma}=p[u \delta]_{\alpha \beta \gamma}+\rho u_{\alpha} u_{\beta} u_{\gamma}-\sum_{i=0}^{18} c_{i \alpha} c_{i \beta} c_{i \gamma} f_{i}^{e q}
$$

The following deviation terms of D3Q19 are obtained with $\theta=p /\left(\rho c_{s}^{2}\right)$

$$
\begin{gathered}
\Psi_{x x x}=\left(\rho u_{x} u_{x} u_{x}+3 p u_{x}\right)-\sum_{i=0}^{18} c_{i x} c_{i x} c_{i x} f_{i}^{e q}=\rho u_{x}\left(\theta-1+u_{x}^{2}\right), \\
\Psi_{x x y}=\left(\rho u_{x} u_{x} u_{y}+p u_{y}\right)-\sum_{i=0}^{18} c_{i x} c_{i x} c_{i y} f_{i}^{e q}=\frac{1}{6} \rho u_{y}\left(\theta-1+3 u_{z}^{2}\right), \\
\Psi_{x y z}=\left(\rho u_{x} u_{y} u_{z}\right)-\sum_{i=0}^{18} c_{i x} c_{i y} c_{i z} f_{i}^{e q}=\rho u_{x} u_{y} u_{z},
\end{gathered}
$$

The deviation terms of $\Psi_{x y y}, \Psi_{y y y}$ etc. can be computed in similarity. Thus the correcting term in D3Q19 lattice set is constructed as follows

$$
\begin{aligned}
\psi_{i}= & \frac{w_{i}}{2 c_{s}^{4}}\left\{\mathcal{H}_{i x x}\left[\frac{\partial}{\partial x} \Psi_{x x x}+\frac{\partial}{\partial y} \Psi_{x x y}+\frac{\partial}{\partial z} \Psi_{x x z}\right]\right. \\
& +\mathcal{H}_{i y y}\left[\frac{\partial}{\partial x} \Psi_{y y x}+\frac{\partial}{\partial y} \Psi_{y y y}+\frac{\partial}{\partial z} \Psi_{y y z}\right] \\
& +\mathcal{H}_{i z z}\left[\frac{\partial}{\partial x} \Psi_{z z x}+\frac{\partial}{\partial y} \Psi_{z z y}+\frac{\partial}{\partial z} \Psi_{z z z}\right] \\
& +2 \mathcal{H}_{i x y}\left[\frac{\partial}{\partial x} \Psi_{x y x}+\frac{\partial}{\partial y} \Psi_{x y y}+\frac{\partial}{\partial z} \Psi_{x y z}\right] \\
& +2 \mathcal{H}_{i x z}\left[\frac{\partial}{\partial x} \Psi_{x z x}+\frac{\partial}{\partial y} \Psi_{x z y}+\frac{\partial}{\partial z} \Psi_{x z z}\right] \\
& \left.+2 \mathcal{H}_{i y z}\left[\frac{\partial}{\partial x} \Psi_{y z x}+\frac{\partial}{\partial y} \Psi_{y z y}+\frac{\partial}{\partial z} \Psi_{y z z}\right]\right\}
\end{aligned}
$$

It is worth noting that only $\Psi_{x x x}, \Psi_{y y y}, \Psi_{z z z}$ is non-zero in D3Q27 lattice set, which exhibit a much simpler correcting term compared with in D3Q19 lattice set. Besides the correcting term of D2Q9 lattice set is the projection of D3Q27 lattice into two-dimensions. 


\section{References}

[1] A. A. Mohamad, Lattice Boltzmann method: fundamentals and engineering applications with computer codes, Springer Science \& Business Media, 2011.

[2] Z. Guo, C. Shu, Lattice Boltzmann method and its applications in engineering, Vol. 3, World Scientific, 2013.

[3] T. Krüger, H. Kusumaatmaja, A. Kuzmin, O. Shardt, G. Silva, E. M. Viggen, The lattice Boltzmann method, Springer International Publishing 10 (2017) 978-3.

[4] L.-S. Luo, W. Liao, X. Chen, Y. Peng, W. Zhang, et al., Numerics of the lattice Boltzmann method: Effects of collision models on the lattice Boltzmann simulations, Physical Review E 83 (5) (2011) 056710.

[5] P. L. Bhatnagar, E. P. Gross, M. Krook, A Model for Collision Processes in Gases. I. Small Amplitude Processes in Charged and Neutral OneComponent Systems, Phys. Rev. 94 (1954) 511-525.

[6] P. Lallemand, L. S. Luo, Theory of the lattice Boltzmann method: Acoustic and thermal properties in two and three dimensions, Physical Review E 68 (2003) 036706.

[7] G. Di Ilio, B. Dorschner, G. Bella, S. Succi, I. Karlin, Simulation of turbulent flows with the entropic multirelaxation time lattice Boltzmann method on body-fitted meshes, Journal of Fluid Mechanics 849 (2018) $35-56$.

[8] N. I. Prasianakis, S. S. Chikatamarla, I. V. Karlin, S. Ansumali, K. Boulouchos, Entropic lattice Boltzmann method for simulation of 
thermal flows, Mathematics and Computers in Simulation 72 (2006) $179-183$.

[9] N. Frapolli, S. Chikatamarla, I. Karlin, Entropic lattice Boltzmann simulation of thermal convective turbulence, Computers \& Fluids.

[10] J. Latt, B. Chopard, Lattice Boltzmann method with regularized precollision distribution functions, Mathematics and Computers in Simulation 72 (2006) 165-168.

[11] K. K. Mattila, P. C. Philippi, L. A. Hegele Jr, High-order regularization in lattice-Boltzmann equations, Physics of Fluids 29 (4) (2017) 046103.

[12] M. Geier, M. Schönherr, A. Pasquali, M. Krafczyk, The cumulant lattice Boltzmann equation in three dimensions: Theory and validation, Computers \& Mathematics with Applications 70 (4) (2015) 507-547.

[13] F. Hajabdollahi, K. N. Premnath, Symmetrized operator split schemes for force and source modeling in cascaded lattice Boltzmann methods for flow and scalar transport, Physical Review E 97 (6) (2018) 063303.

[14] J. Tölke, A thermal model based on the lattice Boltzmann method for low Mach number compressible flows, Journal of Conputational and Therretical Nanoscience 3 (2006) 1-9.

[15] Q. Li, K. H. Luo, Effect of the forcing term in the pseudopotential lattice Boltzmann modeling of thermal flows, Physical Review E - Statistical, Nonlinear, and Soft Matter Physics 89 (5) (2014) 1-7.

[16] X. Nie, X. Shan, H. Chen, Lattice Boltzmann/finite-difference hybrid simulation of transonic flow, AIAA Paper 139 (2009) 2009. 
[17] X. W. Shan, X. F. Yuan, H. D. Chen, Kinetic theory representation of hydrodynamics: a way beyond the Navier-Stokes equation, Journal of Fluid Mechanics 550 (2006) 413-441.

[18] P. Philippi, D. Siebert, L. Hegele Jr, K. Mattila, High-order latticeBoltzmann, Journal of the Brazilian Society of Mechanical Sciences and Engineering 38 (5) (2016) 1401-1419.

[19] N. Frapolli, Entropic lattice Boltzmann models for thermal and compressible flows, Ph.D. thesis, ETH Zurich (2017).

[20] H. Yu, K. Zhao, Lattice Boltzmann method for compressible flows with high Mach numbers, Physical Review E 61 (4) (2000) 3867.

[21] J. Buick, J. Cosgrove, Investigation of a lattice Boltzmann model with a variable speed of sound, Journal of Physics A: Mathematical and General 39 (44) (2006) 13807.

[22] A. Kupershtokh, D. Medvedev, D. Karpov, On equations of state in a lattice Boltzmann method, Computers \& Mathematics with Applications 58 (5) (2009) 965-974.

[23] G. Házi, P. Kávrán, On the cubic velocity deviations in lattice Boltzmann methods, Journal of Physics A: Mathematical and General 39 (12) (2006) 3127.

[24] P. J. Dellar, Lattice Boltzmann algorithms without cubic defects in Galilean invariance on standard lattices, Journal of Computational Physics 259 (2014) $270-283$.

[25] O. Malaspinas, Increasing stability and accuracy of the lattice 
Boltzmann scheme: recursivity and regularization, arXiv preprint arXiv:1505.06900.

[26] Q. Li, K. Luo, Y. He, W. Tao, Couplling lattice Boltzmann model for simulation of thermal flows on standard lattices, Physical Review E 85 (2012) 016710.

[27] Y. Feng, P. Sagaut, W. Tao, A three dimensional lattice model for thermal compressible flow on standard lattices, Journal of Computational Physics 303 (2015) 514-529.

[28] Y. Feng, P. Sagaut, W. Q. Tao, A compressible lattice Boltzmann finite volume model for high subsonic and transonic flows on regular lattices, Computers and Fluids 131 (2016) 45-55.

[29] N. I. Prasianakis, I. V. Karlin, Lattice Boltzmann method for simulation of compressible flows on standard lattices, Physical Review E 78 (1) (2008) 016704.

[30] H. Safari, M. Krafczyk, M. Geier, A lattice Boltzmann model for thermal compressible flows at low mach numbers beyond the Boussinesq approximation, Computers \& Fluids.

[31] L. Fei, K. H. Luo, Cascaded lattice Boltzmann method for thermal flows on standard lattices, International Journal of Thermal Sciences 132 (2018) 368-377.

[32] C. Coreixas, G. Wissocq, G. Puigt, J. Boussuge, P. Sagaut, Recursive regularization step for high-order lattice Boltzmann methods, Phys. Rev. E 96 (2017) 033306. 
[33] J. Jacob, O. Malaspinas, P. Sagaut, A new hybrid recursive regularised bhatnagar-gross-krook collision model for lattice boltzmann methodbased large eddy simulation, Journal of Turbulence 19 (11-12) (2018) 1051-1076.

[34] S. Wilhelm, J. Jacob, P. Sagaut, An explicit power-law-based wall model for lattice-Boltzmann-method-Reynolds-averaged numerical simulations of the flow around airfoils, Physics of Fluids 30 (2018) 065111.

[35] H. Chen, S. A. Orszag, I. Staroselsky, S. Succi, Expanded analogy between Boltzmann kinetic theory of fluids and turbulence, Journal of Fluid Mechanics 519 (2004) 301-314.

[36] Y. Feng, M. Tayyab, P. Boivin, A lattice-Boltzmann model for lowMach reactive flows, Combustion and Flame 196 (2018) 249 - 254.

[37] S. Marié, D. Ricot, P. Sagaut, Comparison between lattice Boltzmann method and Navier-Stokes high order schemes for computational aeroacoustics, Journal of Computational Physics 228 (4) (2009) 1056-1070.

[38] P. Wang, L. Zhu, Z. Guo, K. Xu, A comparative study of LBE and DUGKS methods for nearly incompressible flows, Communications in Computational Physics 17 (3) (2015) 657-681.

[39] S. Chapman, T. G. Cowling, The Mathematical Theory of Non-Uniform Gases, Cambridge University Press, 1970.

[40] K. H. Kim, C. Kim, O.-H. Rho, Methods for the accurate computations of hypersonic flows: I. AUSMPW+ scheme, Journal of Computational Physics 174 (1) (2001) 38-80. 
[41] C. Hirsch, Numerical computation of internal and external flows: The fundamentals of computational fluid dynamics, Elsevier, 2007.

[42] Y.-L. Feng, S.-L. Guo, W.-Q. Tao, P. Sagaut, Regularized thermal lattice Boltzmann method for natural convection with large temperature differences, International Journal of Heat and Mass Transfer 125 (2018) $1379-1391$.

[43] H. Lamb, Hydrodynamics, Cambridge university press, 1993.

[44] H. Tryggeson, Analytical vortex solutions to navier-stokes equation, Ph.D. thesis, Växjö University Press (2007).

[45] H. Tryggeson, M. D. Lyberg, Stationary vortices attached to flat roofs, Journal of Wind Engineering and Industrial Aerodynamics 98 (1) (2010) 47-54.

[46] C. K. Tam, J. C. Webb, Dispersion-relation-preserving finite difference schemes for computational acoustics, Journal of computational physics 107 (2) (1993) 262-281.

[47] M. Dumbser, E. F. Toro, On universal Osher-type schemes for general nonlinear hyperbolic conservation laws, Communications in Computational Physics 10 (3) (2011) 635-671.

[48] M. Tavelli, M. Dumbser, A pressure-based semi-implicit space-time discontinuous Galerkin method on staggered unstructured meshes for the solution of the compressible Navier-Stokes equations at all Mach numbers, Journal of Computational Physics 341 (2017) 341-376.

[49] D. R. Chapman, Temperature and velocity profiles in the compressible 
laminar boundary layer with arbitrary distribution of surface temperature, Journal of the Aeronautical Sciences 16 (9) (1949) 547-565.

[50] D. De Grazia, D. Moxey, S. Sherwin, M. Kravtsova, A. Ruban, Direct numerical simulation of a compressible boundary-layer flow past an isolated three-dimensional hump in a high-speed subsonic regime, Physical Review Fluids 3 (2) (2018) 024101.

[51] F. M. White, I. Corfield, Viscous fluid flow, Vol. 3, McGraw-Hill New York, 2006. 